\title{
Radiological Anatomy of Stomach and Duodenum with Clinical Significance
}

\author{
Zainab Vora ${ }^{1, \odot ~}$ Ankur Goyal ${ }^{1, \oplus}$ Raju Sharma ${ }^{1}$ \\ ${ }^{1}$ Department of Diagnostic and Interventional Radiology, All India \\ Institute of Medical Sciences, New Delhi, India
}

\begin{abstract}
Address for correspondence Zainab Vora, MD, Department of Diagnostic and Interventional Radiology, All India Institute of Medical Sciences, New Delhi, India

(e-mail: zainab8@gmail.com).
\end{abstract}

\begin{abstract}
- Keywords:

- stomach

- duodenum

- radiological anatomy

- imaging modalities

Anatomy is the key to accurate imaging interpretation. It is essential for radiologists to thoroughly understand the normal anatomy and spatial relationships of the stomach and duodenum to accurately localize the site of abnormality. In this article, we describe in detail the gross and applied radiological anatomy of the stomach and duodenum, and the current role of various radiological investigations (including barium studies, endoscopic ultrasonography, computed tomography, and magnetic resonance imaging) in the evaluation of the diseases involving the stomach and duodenum.
\end{abstract}

\section{Introduction}

Diseases of the stomach and duodenum are a frequent but potentially underrated cause of hospital visits. These include a wide spectrum ranging from functional dyspepsia and benign conditions (like gastroesophageal reflux disease, gastritis, duodenitis) to life-threatening conditions (including malignancy, perforation, obstruction, and hemorrhage). Much akin to the disease spectrum, there is a wide gamut of investigations available for the evaluation of the stomach and duodenum. Upper gastrointestinal (GI) endoscopy is the investigation of choice for evaluation of gastric diseases; however, it has various limitations: it is invasive, not tolerated by many patients, and may lead to erroneous interpretation in diseases like large hiatal hernia, gastric volvulus, submucosal lesions, and evaluation of the third part of the duodenum. ${ }^{1}$ For these reasons, radiological modalities are commonly used since they provide comprehensive luminal and extraluminal information noninvasively. Thus, it is imperative to know the radiological anatomy of the stomach and duodenum as well as understand the spatial relationships on various imaging investigations.

published online

May 3, 2021
DOI https://doi.org/

$10.1055 / \mathrm{s}-0041-1727579$ ISSN 2581-9933

\section{Embryology}

During the fourth week of development, the stomach develops as an expanding fusiform dilation of the caudal part of the foregut. By the sixth week, the dorsal aspect of the stomach grows faster than the ventral aspect, resulting in the formation of the characteristic curvatures. As it enlarges, it rotates 90 degrees clockwise along its longitudinal axis and anteroposterior (AP) axis pulling the pyloric region upward and to the right, while the cardia shifts to the left and slightly downward. ${ }^{2}$ Then the stomach assumes its final position with its long axis running from above left to below right.

\section{Gross Anatomy}

The stomach is the dilated part of the GI tract and has three main functions: storage of food (in adults, it has a capacity of $~ 1.5$ liters), mixing food with enzymes to form semisolid chyme, and releasing the chyme at a controlled rate to the lower GI tract. It lies in the left upper abdomen, extending from the left costal margin and dome of the diaphragm above to the umbilicus below. ${ }^{3}$ When fully distended, it forms a
(C) 2021. Indian Society of Gastrointestinal and Abdominal Radiology. This is an open access article published by Thieme under the terms of the Creative Commons Attribution-NonDerivative-NonCommercial-License, permitting copying and reproduction so long as the original work is given appropriate credit. Contents may not be used for commercial purposes, or adapted, remixed, transformed or built upon. (https://creativecommons.org/licenses/by-nc-nd/4.0/). Thieme Medical and Scientific Publishers Pvt. Ltd. A-12, 2nd Floor, Sector 2, Noida-201301 UP, India 
"J"-shaped configuration with two openings, the cardiac (proximally) and pyloric (distally) orifices; two borders, the greater and lesser curvature; and two surfaces, anterosuperior and posteroinferior. This muscular cavity is relatively fixed at both ends but the rest is quite mobile. Much of it lies protected by the left lower ribs. These factors play an important role in protecting the stomach in blunt trauma to the abdomen; however, the large size makes it susceptible to injury by penetrating trauma.

It is divided into the following parts (-Fig. 1):

- Cardia: The area into which the esophagus opens, with a physiological sphincter mechanism to prevent reflux.

- Fundus: Dome-shaped, superolateral part of the stomach, located above and to the left of the cardia.

- Body: Extends from the cardia to the level of incisura angularis, a small anatomical notch on the stomach located on the lesser curvature of the stomach, which marks the junction between the body and the antrum.

- Pyloric Antrum: From incisura angularis to pyloric opening.

Pylorus: Tubular part of the stomach around $2.5 \mathrm{~cm}$ long with a thick muscular wall, forming the pyloric sphincter, controlling the outflow of contents from the stomach.

The stomach is completely covered by peritoneum; lesser curvature forming the right (concave) border of the stomach is suspended from the liver by the lesser omentum, while the greater curvature forming the much longer and convex left border is suspended from the spleen by the gastrosplenic ligament superiorly and from the transverse colon by the greater omentum. ${ }^{4}$

The anterior surface of the stomach is in relation to the left dome of the diaphragm, anterior abdominal wall, and the caudate lobe of the liver. The remaining posterior part of the stomach is referred to as "stomach bed," which is related to the left adrenal gland, the upper pole of the left kidney, splenic artery, pancreas, transverse mesocolon, and occasionally the transverse colon.

The stomach is composed of four layers: columnar mucosal epithelium, which is thick and vascular, with multiple folds in the longitudinal direction, called rugae, which flatten out upon distension allowing an increase in efficiency; submucosa; muscularis propria layer, consisting of inner oblique, middle circular, and outer longitudinal layers (unlike other GI organs, which only have two layers); and the outer serosal layer, continuous with the peritoneum. Parietal cells, which produce hydrochloric acid and intrinsic factor, and chief cells, which produce pepsin precursors, are found in the fundus and body. Gastrin is produced in the antrum.

The duodenum is a " $\mathrm{C}$ "-shaped tube, $25 \mathrm{~cm}$ in length,

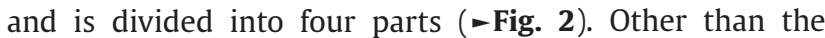
duodenal "cap," the rest of the parts of the duodenum are retroperitoneal. ${ }^{5}$

First part (Duodenal “cap”): The first (superior) portion, $2.5 \mathrm{~cm}$ in length, begins at the pylorus and runs posterosuperiorly on the transpyloric plane at the level of L1. It is the only part of the duodenum that is covered by the peritoneum, with the lesser omentum attached to the superior border and the greater omentum attached to the inferior border. The lesser sac lies posterior to D1. The gastroduodenal artery runs inferiorly behind the first part of the duodenum, an important landmark, as an ulcer on the posterior wall will erode this artery leading to massive bleeding.

An important diagnostic dilemma wherein a perforated duodenal ulcer may mimic acute appendicitis is when a duodenal ulcer on the anterior wall of the first part perforates into the greater sac above the transverse colon, which directs it into the right paracolic gutter.

Second part: This portion runs vertically downward along with L2 and L3 vertebrae, lateral to the head of the pancreas. Posteromedially lies the opening of the duodenal papilla
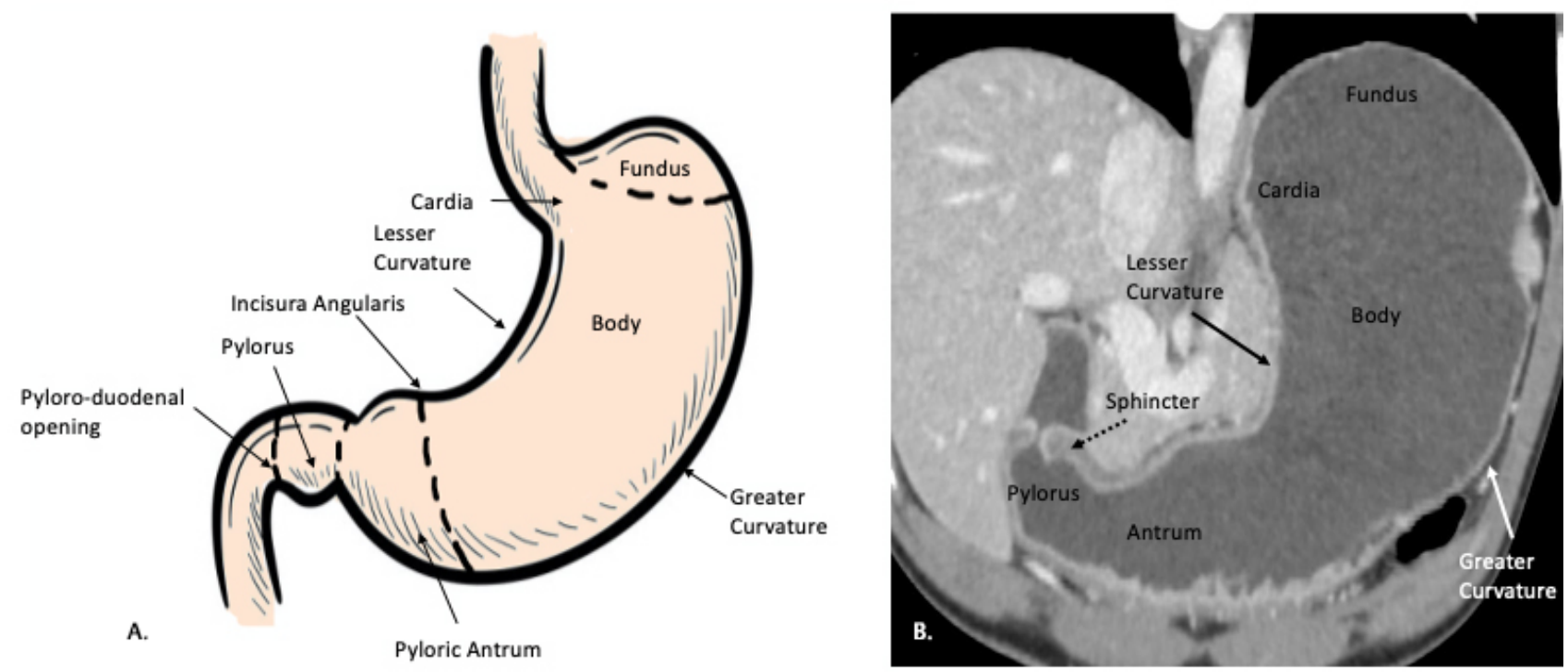

Fig. 1 Gastric anatomy. (A) Schematic illustration. (B) Coronal contrast-enhanced computed tomography (CT) image of the normal stomach with oral water demonstrates the fundus, body, antrum, and pylorus of stomach. The pyloric sphincter is well visualized on the CT image (dotted arrow). 


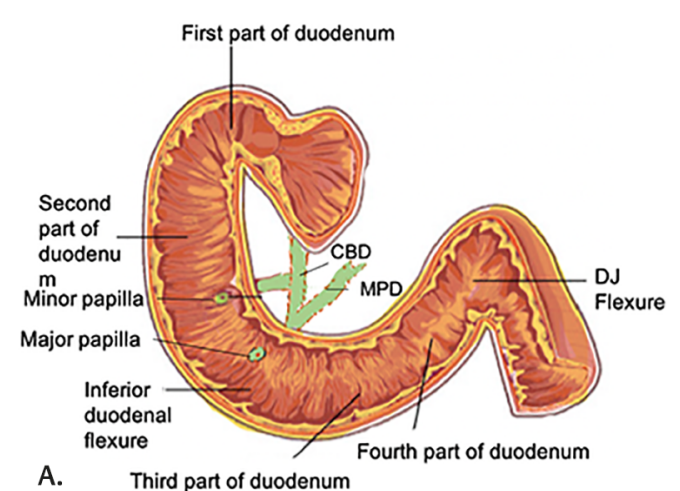

A. Third part of duodenum
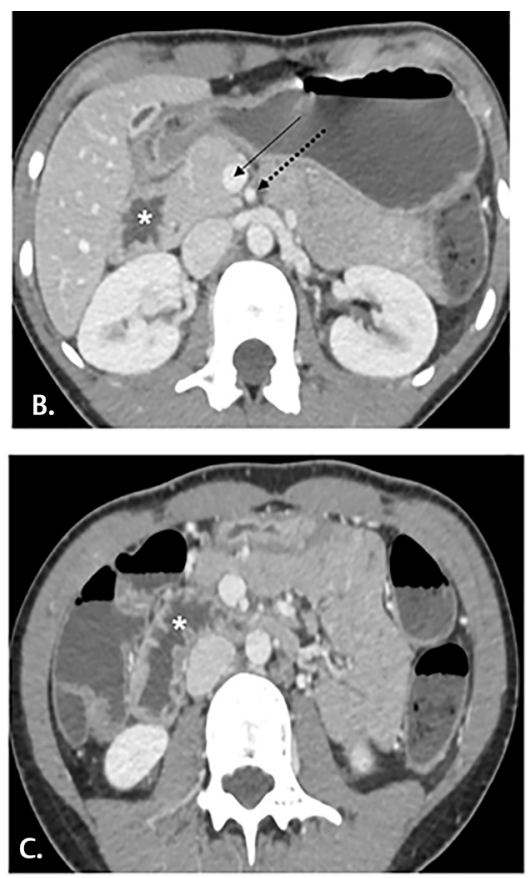

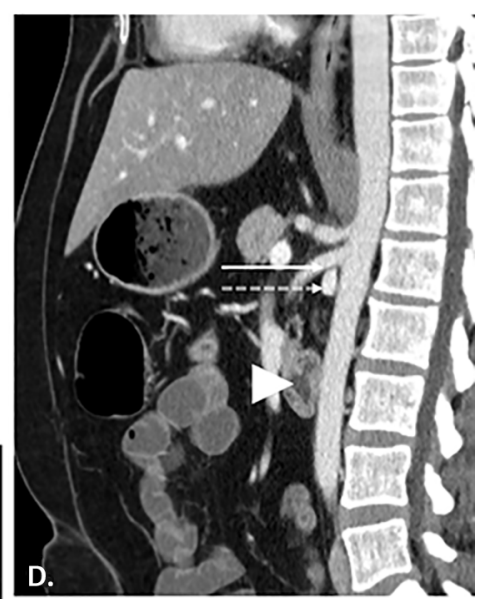

Fig. 2 Anatomy of duodenum. (A) Schematic diagram showing the anatomy of duodenum with openings of the common bile duct (CBD) and main pancreatic duct in the major papilla and the accessory pancreatic duct in the minor papilla in the second part of duodenum. (B, C) Axial contrast-enhanced computed tomography (CECT) with oral water demonstrates the relationship of D2 and D3 $\left({ }^{*}\right)$ with pancreas and superior mesenteric artery (SMA: dotted arrow) and superior mesenteric vein (solid arrow) medially. (D) Sagittal CECT with oral water demonstrates the relation of left renal vein (dotted arrow) and third part of duodenum (arrowhead) at the angle of aorta and SMA (solid arrow). This explains the pathophysiology of compression of left renal vein and D3 by reduced aortomesenteric angle in nutcracker syndrome and SMA syndrome, respectively DJ, duodenojejunal; MPD, main pancreatic duct.

or ampulla of Vater or major duodenal papilla, while the minor/accessory pancreatic duct opens a little higher on the minor duodenal papilla.

Third part: It runs horizontally to the left, below the inferior margin of the head of the pancreas. In severe crush injuries, the third part of the duodenum is crushed against the L3 vertebra, as most of the duodenum is rigidly fixed to the posterior abdominal wall by the peritoneum, and cannot move away from crush injuries.

Fourth part: The fourth part of the duodenum runs cephalad and to the left of midline, lying medial to the left psoas muscle, fixed by the ligament of Treitz (a peritoneal fold that is attached to the right crus of the diaphragm). This marks the superior end of the root of the mesentery. This is an important anatomical landmark, particularly important for the diagnosis of malrotation of the bowel, where this fourth part is not seen crossing the midline. Close to the duodenojejunal (DJ) junction, there are four pocket-like pouches of peritoneum called the superior, inferior, para, and retroduodenal recesses, which play an important role in the etiopathogenesis of internal hernias.

Histologically the duodenum is similar to the rest of the GI tract: mucosa with columnar epithelium, lamina propria, submucosa, and muscularis layer consisting of an inner circular and outer longitudinal layer. Except for the first part, the duodenum has circular folds of the mucosa and submucosa, called the valves of Kerckring, also called plicae circulares or valvulae conniventes that increase the absorption area of the duodenum up to 1,500 times. A characteristic feature of the duodenum is the Brunner's glands embedded in the submucosa, which produces mucous secretions rich in bicarbonate.

\section{Arterial Supply}

The arterial supply to the stomach is derived from the branches of the celiac artery, which have an extensive anastomotic network between them. ${ }^{3}$

The celiac axis (-Fig. 3) divides into left gastric, hepatic, and splenic arteries. The hepatic artery gives rise to the right gastric and the gastroduodenal arteries. The gastroduodenal artery gives rise to the right gastroepiploic artery.

The left gastric artery, a branch of the celiac axis, runs cranially from the origin and descends along and supplies the superior aspect of lesser curvature, while the right gastric artery, a branch of the hepatic artery, supplies the inferior aspect of the lesser curvature. The left gastroepiploic artery, a branch of the splenic artery, supplies the superior part of the greater curvature whereas the right gastroepiploic artery, a branch of the gastroduodenal artery, supplies the inferior portion.

Short gastric arteries, branches from the splenic artery, supply the fundus via the gastrosplenic ligament.

The duodenum is predominantly supplied by two arteries: the superior and inferior pancreaticoduodenal arteries. The gastroduodenal artery descends behind the first part of the duodenum and divides into the right gastroepiploic and the superior pancreaticoduodenal branches. The superior pancreaticoduodenal artery supplies the proximal first 


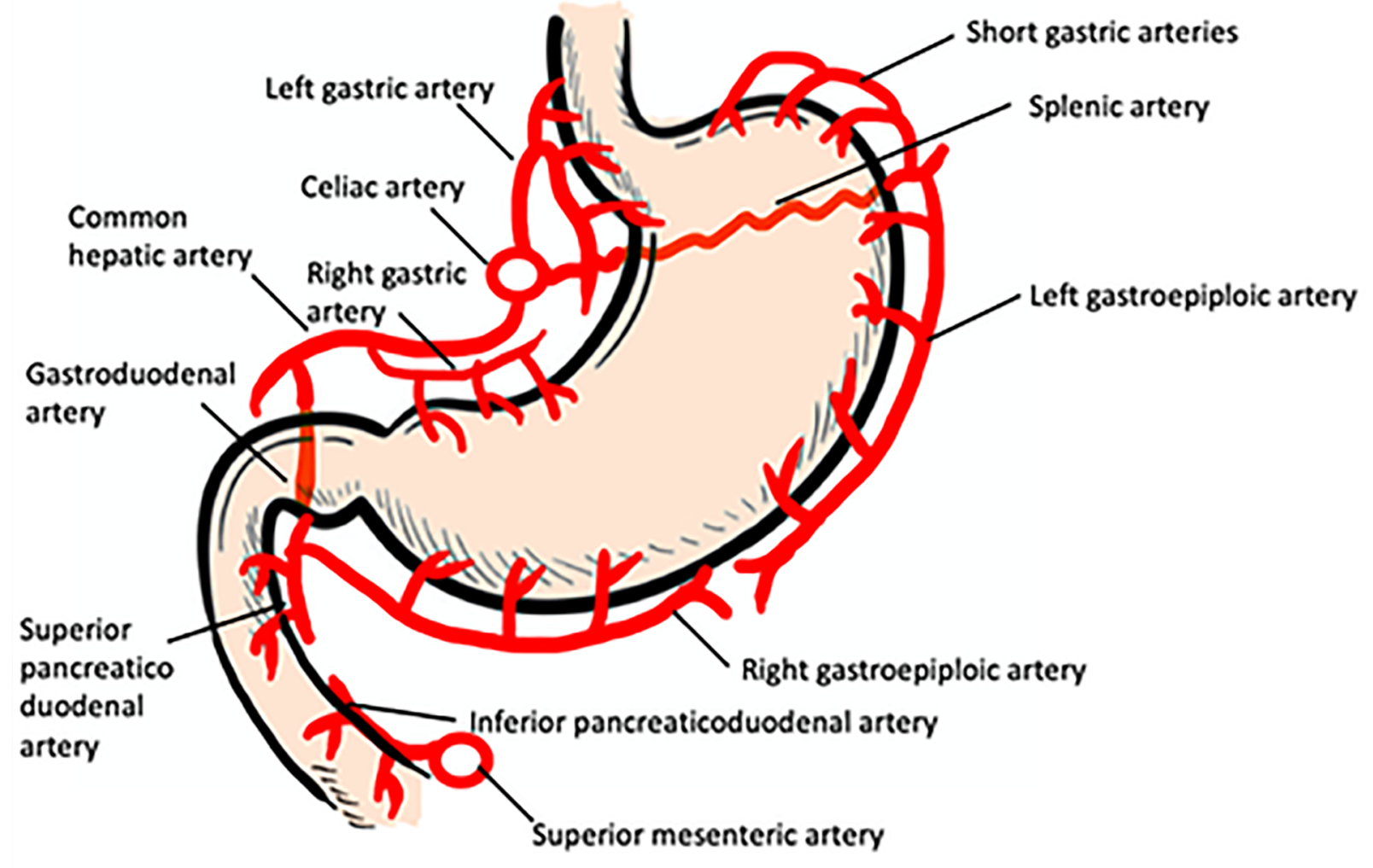

Fig. 3 Schematic illustration showing the arteries supplying the stomach and duodenum. The right and left gastric arteries supplying the lesser curvature, and right and left gastroepiploic arteries supplying the greater curvature arising from the celiac artery. The superior and inferior pancreaticoduodenal arteries supply the duodenum.

and second parts of the duodenum up to the major duodenal ampulla, while the third and fourth parts of the duodenum is supplied by the inferior pancreaticoduodenal artery, the first branch of the superior mesenteric artery.

\section{Venous Drainage}

The veins of the stomach directly drain into the portal venous system through the right and left gastric veins, while the short gastric and the left gastroepiploic veins join the splenic vein. The right gastroepiploic vein drains into the superior mesenteric vein. Two veins following the arteries, superior and inferior pancreaticoduodenal veins, drain into the portal vein and superior mesenteric vein, respectively. ${ }^{3}$

\section{Lymphatic Drainage}

The lymphatic drainage of the stomach and proximal duodenum follows the arteries into the left and right gastric nodes, left and right gastroepiploic nodes, short gastric nodes, and gastroduodenal nodes. All the gastric lymphatics eventually drain into the celiac lymph nodes, located around the root of the celiac artery. The D2 and D3 parts of the duodenum drain into the pancreaticoduodenal nodes to the superior mesenteric nodes at the origin of the superior mesenteric artery. ${ }^{4}$

Intraoperative anatomy of stomach and duodenum is illustrated in - Fig. 4.

\section{Imaging Modalities and Applied Anatomy}

\section{Radiograph}

The stomach is not well visualized on the radiographs. However, the gastric bubble, representing the gas outlining the fundus of the stomach, is often visible on an erect chest or abdominal radiograph.

\section{Barium Meal Studies}

Endoscopy remains the gold standard for the evaluation of the lumen of the stomach and duodenum. With its increased availability, there has been a marked decline in the numbers of conventional upper GI barium studies. ${ }^{6}$ However, it has still not passed into history and conventional barium studies continue to be used as initial noninvasive diagnostic studies in patients with dysphagia. These may also be done for patients presenting with dyspepsia, weight loss, anemia, or an upper abdominal mass, though computed tomography (CT) is more commonly done. Hence it is important to understand the basic principles of these studies. A double-contrast barium meal is preferred over a single-contrast study as it provides greater mucosal detail with optimal distension and mucosal coating. However, a double-contrast examination requires more training in the technical aspects of the study as well as interpretation (-Fig. 5).

Technique: As food or fluids will hinder proper mucosal coating, patients are instructed to fast overnight before 

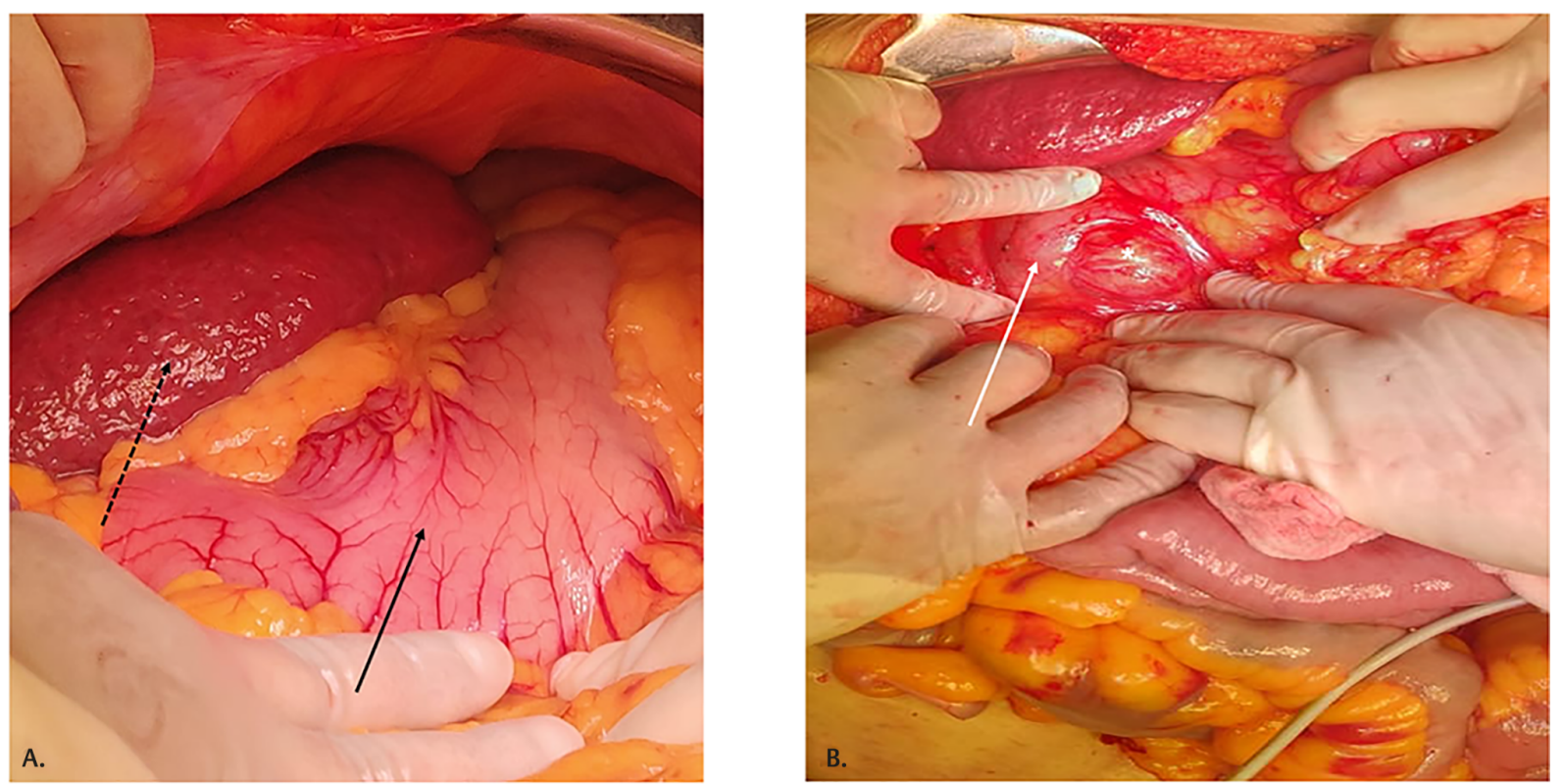

Fig. 4 Intraoperative anatomy of stomach and duodenum. (A) Intraoperative image showing the external surface of stomach (solid arrow) and omentum. Note the cirrhotic liver (dotted arrow). (B) Intraoperative image showing second part of duodenum (arrow) with a diverticulum (asterisk).
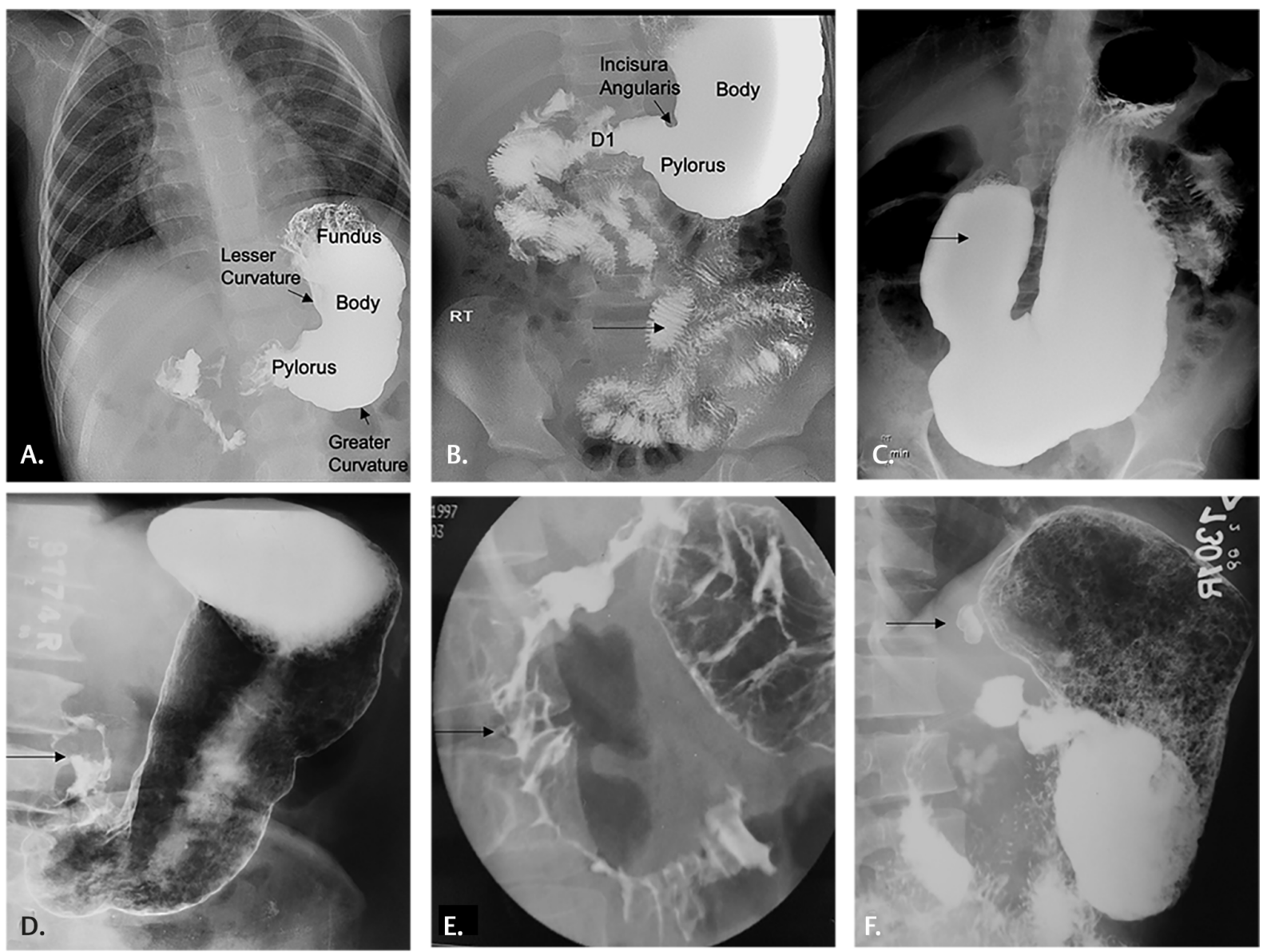

Fig. 5 Barium meal showing fluoroscopic appearance of stomach and duodenum. (A, B) Barium meal and follow-through images showing the normal anatomy of stomach and proximal small bowel. Note the valvulae conniventes in the jejunum loops (arrow). (C) Single-contrast barium meal image in a case of gastric outlet obstruction showing overdistended stomach up to the pylorus (arrow). (D) Double-contrast barium meal spot showing irregular narrowing (arrow) of the distal part of pyloric antrum in a case of adenocarcinoma. (E) Double-contrast barium meal spot showing long-segment nodular-fold thickening with thin streak of barium in lumen of first and second parts of duodenum (arrow) in a case of lymphoma. (F) Double-contrast barium meal spot showing a well-defined saccular outpouching from the gastric wall (arrow), consistent with a gastric diverticulum. 
the study, and avoid smoking as it increases gastric secretions and impairs mucosal coating. Single-contrast upper GI study involves drinking 50 to $70 \% \mathrm{w} / \mathrm{v}$ barium suspension (after diluting the commercially available $95 \% \mathrm{w} / \mathrm{v}$ preparation). The double-contrast barium meal examination typically includes the following elements: good mucosal coating, adequate gaseous distention, and positional maneuvers. The patient is given effervescent sodium bicarbonate or dimethicone granules, a gas-producing agent, following which the patient drinks the $200 \% \mathrm{w} / \mathrm{v}$ of barium suspension (300 gm barium powder is mixed with $70 \mathrm{~mL}$ of water to get $150 \mathrm{~mL}$ of high-density low-viscosity suspension [200\% $\mathrm{w} / \mathrm{v}]$ ). Some centers also use hypotonic agents like Buscopan or Glucagon to decrease peristalsis and achieve better gastric distension. $^{7}$

The left posterior oblique ( \pm AP and lateral) view of the esophagus is taken at the beginning, followed by supine and right oblique views to check for reflux. If reflux is not elicited, maneuvers like coughing, swallowing water, or a Trendelenburg position may be attempted. Following this, the patient is asked to lie down in the left decubitus position, which prevents rapid filling of the distal duodenum. Next, under fluoroscopy, the patient is asked to turn to the right side and then quickly prone, to the left, and supine again, finishing in a right anterior oblique position (completing a circle), until a good coating of the stomach is achieved. The gastric mucosa is characterized by two features, the areae gastricae, which form the mucosal surface pattern, and the gastric rugae, which form the gross pattern. The visualization of areae gastricae pattern on double-contrast studies is considered a marker of the adequate mucosal coating. It appears as a reticular network of contrast filling the grooves between the mucosal tufts. Gastric rugae are approximately 3 to $5 \mathrm{~mm}$ thick smooth mucosal and submucosal folds, along the long axis of the stomach. ${ }^{8}$ When the barium column enters the duodenum, the patient is turned to the right anterior oblique position and compression spots are taken. In postoperative cases, oral contrast studies are done to detect leaks and assess the movement of the contrast column across the anastomotic site. The safest contrast in these situations is nonionic iodinated contrast. Katz criteria ${ }^{9}$ are used to determine subtle abnormalities indicative of intestinal malrotation, by assessing nine findings on upper GI contrast studies ( - Table $\mathbf{1}$ ).

The following fluoroscopic spots are taken for the different parts of the stomach and duodenum: ${ }^{10-12}$

- Right anterior oblique: Antrum and greater curvature; duodenum C-loop

- Right lateral: Gastric cardia; fundus

- Supine: Body and antrum; DJ flexure

- Left anterior oblique: Lesser curvature en face

- Prone: Duodenal cap

- Erect: Fundus

- Lateral: DJ flexure

\section{Ultrasound}

While endoscopy is the gold standard for the investigation of the stomach and upper GI tract, most patients are initially
Table 1 Katz criteria for the assessment of intestinal malrotation

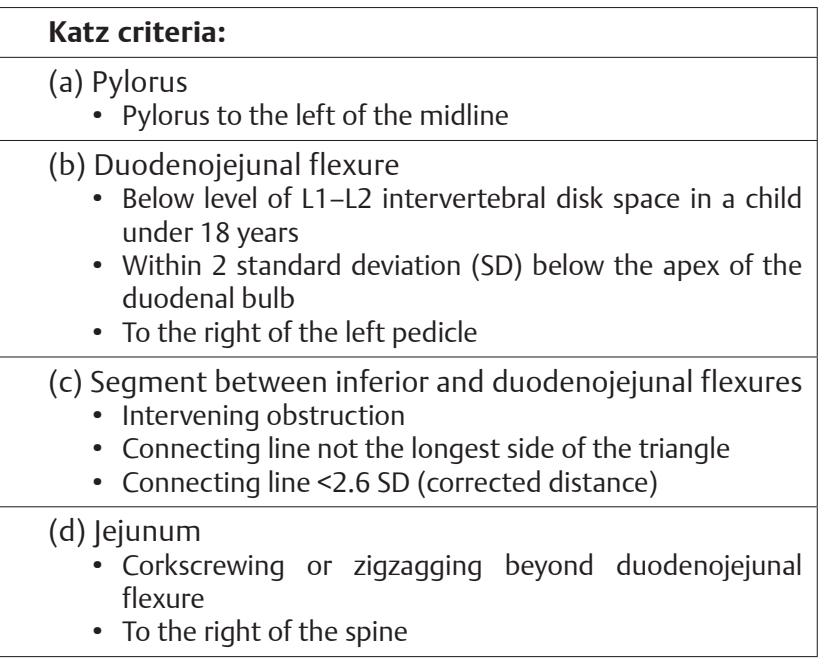

Note: One abnormality represents normal variation; two abnormalities represent indeterminate finding; three and more abnormalities found together indicate malrotation.

investigated by an ultrasound (US) of the abdomen. The literature reporting stomach findings in transabdominal US are minimal, but, using a dedicated protocol, stomach pathologies may be diagnosed on the US. ${ }^{13}$ Patients should be fasting for at least 6 hours and asked to drink 400 to $500 \mathrm{~mL}$ of water immediately before imaging, as quickly as possible to minimize ingestion of air. The pylorus is the easiest part to be located and examination should start from there. The probe can then be held at an oblique angle at the left costal margin to assess the body and fundus. Following this, the transverse epigastric view will help visualize the long axis of the stomach. ${ }^{14}$ The stomach wall appears as three discrete layers on transabdominal US with hyperechoic mucosa, hypoechoic muscularis, and hyperechoic serosal layer. Abnormal wall thickening, presence of solid or cystic masses, or presence of thickened pyloric musculature in congenital hypertrophic pyloric stenosis are well visualized. ${ }^{15}$

\section{Computed Tomography}

Endoscopy and barium meal give valuable information about the lumen but have a limited role in assessing the mural and extramural lesions. CT provides comprehensive information, not only about the stomach and duodenum but also adjacent structures, and is especially useful in the diagnosis and staging of tumors of the stomach and duodenum. ${ }^{16}$ Recent advances in multidetector CT with thin collimation allow near-isotropic multiplanar reconstructions allowing early detection of various diseases, including the subtle mural abnormalities. Patient preparation includes no solid food for at least 6 hours. To optimize the assessment with CT, the stomach and duodenum must be optimally distended. This is usually performed by asking the patient to drink 500 to $750 \mathrm{~mL}$ of water 15 to 20 minutes before the procedure. Water is preferred over positive oral contrast so that even the subtle abnormal wall enhancement can be detected. Negative contrast such as air, produced by effervescent gas-producing 
crystals, may also be used. Routinely, a single porto-venous phase acquisition is performed 60 to 70 seconds after the start of intravenous contrast administration. To optimize visualization, the patients with suspected lesions in the cardiac or fundus region may be imaged in the supine or left lateral decubitus position, and lesions in the distal two-thirds, in prone or $30^{\circ}$ right posterior oblique position. ${ }^{17,18}$

With adequate distension, the gastric wall (normally up to $3 \mathrm{~mm}$ thick) can be readily evaluated with the mucosal surface appearing as a thin hyperdense layer with an isodense muscularis layer (-Table 2).

Greater and lesser curvatures are better visualized in coronal and sagittal images while in the axial plane, anterior

Table 2 Computed tomography technique for examination of the stomach

\begin{tabular}{|l|}
\hline Patient preparation and positioning \\
\hline - Fasting: No solid food for at least $6 \mathrm{~h}$ \\
\hline - Oral contrast: $500-750 \mathrm{~mL}$ of neutral contrast water or fla- \\
vored methylcellulose preparation \\
\hline - Hypotonia: $20 \mathrm{mg}$ Buscopan intravenously \\
\hline - Patient position: Supine; prone position preferred if known \\
lesion in the antrum or pyloric wall \\
\hline Scanning technique \\
\hline - Coverage: Diaphragm to pubic symphysis \\
\hline - Contrast injection: $1.5 \mathrm{~mL} / \mathrm{kg}$ body weight followed by $50 \mathrm{~mL}$ \\
saline at 3 mL/sec \\
\hline - Arterial phase: 30 sec only for tumor staging or suspected \\
arterial bleed \\
\hline - Porto-venous phase: $60-70$ sec in all cases \\
\hline - Slice collimation: $1.25 \mathrm{~mm}$ \\
\hline - Computed tomography dose index: 8 -12 mGy \\
\hline - Reconstructions: Multiplanar, $1-1.5$ mm reconstructions \\
\hline
\end{tabular}

and posterior surfaces are better descriptors. Pathological changes in the stomach are demonstrated by localized or generalized thickening of either the mucosal or mural layers or both. Pylorus appears mildly thickened compared to the rest of the stomach because of greater muscle thickness. Discrete masses, both endo- and exoluminal, are well visualized, with an assessment of the extent, lymphadenopathy, as well as distant metastases. With the advent of the three-dimensional volumetric rendering technique, surface-shaded volume-rendered images can be generated, similar to the conventional barium images. Endoluminal fly-through view, called virtual gastroscopy, may be performed following the administration of gas-forming granules orally, and intravenous contrast media simulate the images obtained at endoscopy and allow mucosal evaluation. ${ }^{19-21}$

\section{Magnetic Resonance Imaging}

Magnetic resonance imaging (MRI) of the stomach is not routinely performed due to the inherent artifacts from peristalsis and respiration (-Fig. 6). Only a few studies have been performed to study the role of MRI in gastric diseases, as conventional MRI does not provide the very high spatial resolution needed to evaluate subtle gastric wall pathologies.22,23 MRI endoscopy, ${ }^{24,25}$ a new technique evaluated initially for rectal carcinoma, wherein a radiofrequency coil is mounted at the end of an endoscope, has gained recent interest to depict the anatomical layers of gastric walls and has shown promising results.

\section{Endoscopic Ultrasonography}

Endoscopy is the investigation of choice to visualize mucosal diseases. When combined with high-frequency US, there is a unique opportunity to visualize the bowel wall with high spatial resolution, as well as perform targeted endoscopic biopsy. The US probe is mounted on the tip of a flexible gastroscope that can be targeted to the area of interest, without

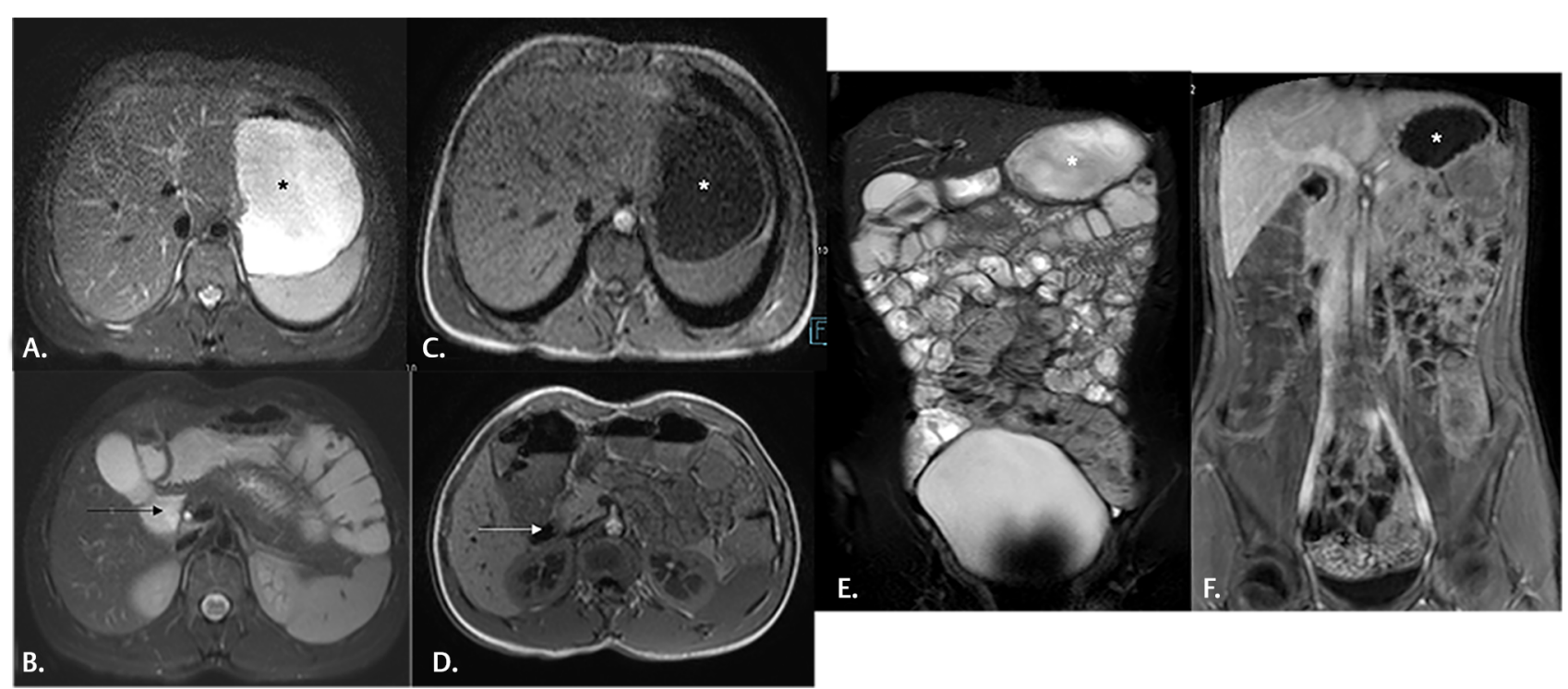

Fig. 6 Magnetic resonance imaging (MRI) appearance of stomach and duodenum. Axial T2 (A, B), axial T1 (C, D), coronal balanced turbo field echo $(\mathbf{E})$, and coronal post-Gd T1 (F) showing the normal appearances and relations of the body of well-distended stomach $\left({ }^{*}\right)$ and the second part of duodenum (solid arrows). Conventional MRI images usually do not show the different layers of gastric wall, but abnormal thickening can be detected in a well-distended stomach. 


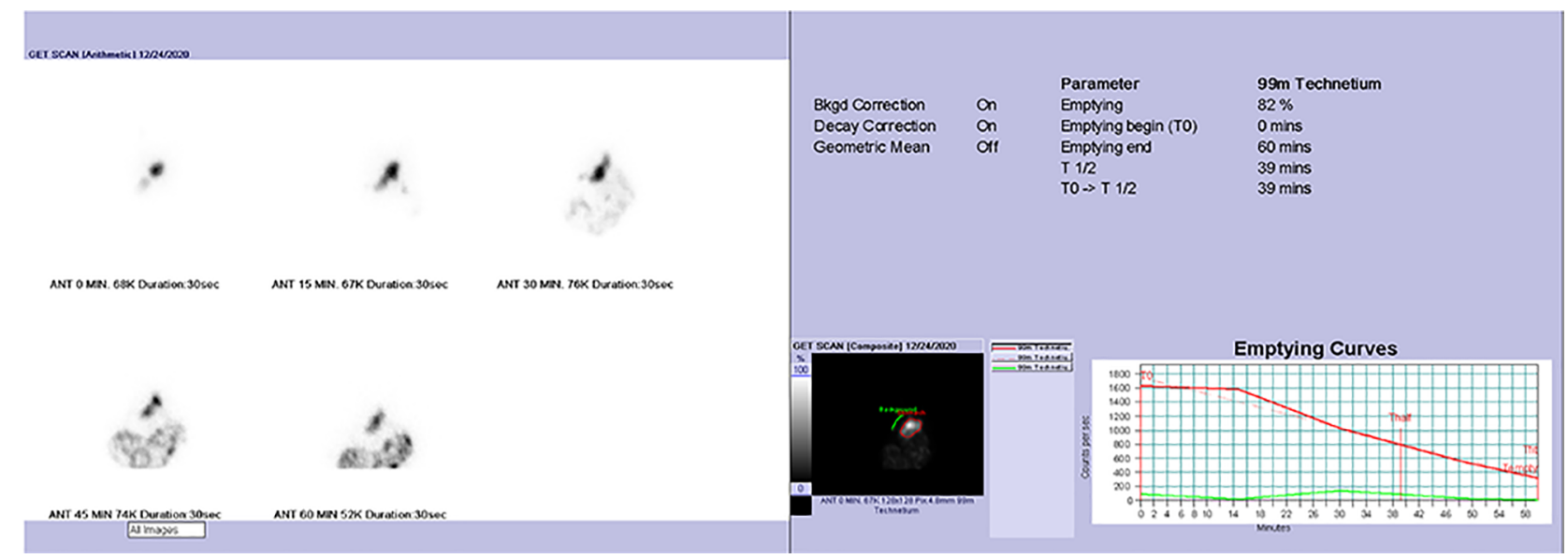

Fig. 7 Gastric emptying test. Image showing a normal gastric emptying test done using a solid meal (performed in our setting using idli labeled with Technitium-99m). Anterior static images are acquired at $0,15,30,45$, and 60 minutes after ingestion, and the emptying curves demonstrate a normal T1/2 of 30 to 90 minutes.

interference from intervening bowel gas to produce good quality images. ${ }^{26}$ During the examination, the stomach is filled with 500 to $700 \mathrm{~mL}$ of water to produce an acoustic window. The limitations include lack of routine availability, operator dependence, and cost. Standard endoscopic US images demonstrate the five layers of the gastric wall; alternating hypoechoic and hyperechoic bands correspond to the following five anatomic wall layers: superficial mucosa (hyperechoic), deep muscularis mucosa (hypoechoic), submucosa (hyperechoic), muscularis propria (hypoechoic), and serosa (hyperechoic). The endoscopic US provides accurate information regarding the depth of mural invasion, the extension of the lesion, and adjacent lymphadenopathy. ${ }^{27}$

\section{Radionuclide Studies}

Gastric emptying scintigraphy is considered to be the gold standard investigation for the assessment of gastric motility. It is used to evaluate patients suspected to have rapid or delayed gastric emptying. Gastric emptying is a physiological process controlled by a complex interplay of the physical and chemical composition of the meal, sympathetic and parasympathetic innervation, and circulating neurotransmitters. The standard imaging protocol involves measuring the rate of transit of radiolabeled solid food (commonly $0.5-1 \mathrm{mCi}$ of Tc99m sulfur colloid in a mixture of approximately $120 \mathrm{~g}$ of egg whites or idli in India) through the stomach. ${ }^{28}$ Using a modern dual-head camera system, a series of images is obtained in the anterior and posterior projections immediately upon ingestion, and then repeated at hourly intervals of 1,2 , and 4 hours. The data are displayed as a time-activity curve using geometric mean counts derived from the anterior and posterior projections. A study is considered to have delayed gastric emptying if there is more than $60 \%$ of the solid meal remaining at 2 hours or more than $10 \%$ of the meal remaining at 4 hours $^{29}$ ( - Fig. 7).

\section{Conclusion}

A variety of pathologic conditions involving the stomach and duodenum may demonstrate a myriad of radiologic features.
Multidetector CT is the current workhorse for the radiological evaluation of gastric and duodenal diseases, complemented by endoscopy for luminal information. Understanding the anatomical facts and relationships on various investigations is crucial to localize the disease and provide an accurate diagnosis.

\section{Note}

Institutional Review Board has approved the study.

\section{Funding}

None.

\section{Conflict of Interest}

None declared.

\section{Acknowledgment}

The authors would like to thank Dr. S.A. Shamim, Associate Professor, Department of Nuclear Medicine, AIIMS, New Delhi.

\section{References}

1 Laufer I, Double Contrast Gastrointestinal Radiology with Endoscopic Correlation. Philadelphia: Saunders;1979 1-8

2 McCracken KW, Wells JM. Mechanisms of embryonic stomach development. Semin Cell Dev Biol 2017;66:36-42

3 Snell RS. Clinical Anatomy for Medical Students. Boston: Little, Brown;1995

4 Soybel DI. Anatomy and physiology of the stomach. Surg Clin North Am 2005;85(5):875-894

5 Gelberg HB. Comparative anatomy, physiology, and mechanisms of disease production of the esophagus, stomach, and small intestine. Toxicol Pathol 2014;42(1):54-66

6 Op den Orth JO. Use of barium in evaluation of disorders of the upper gastrointestinal tract: current status. Radiology 1989;173(3):601-608

7 Stevenson GW, Somers S, Virjee J. Routine double-contrast barium meal: appearance of normal duodenal papillae. Diagn Imaging 1980;49(1):6-14

8 Ominsky SH, Margulis AR. Radiographic examination of the upper gastrointestinal tract: a survey of current techniques. Radiology 1981;139(1):11-17

9 Katz ME, Siegel MJ, Shackelford GD, McAlister WH. The position and mobility of the duodenum in children. AJR Am J Roentgenol 1987;148(5):947-951 
10 Evers K, Kressel HY. Principles of performance and interpretation of double-contrast gastrointestinal studies. Radiol Clin North Am 1982;20(4):667-685

11 Levine MS, Rubesin SE, Herlinger H, Laufer I. Double-contrast upper gastrointestinal examination: technique and interpretation. Radiology 1988;168(3):593-602

12 Chapman S, Nakielny R, A Guide to Radiological Procedures. 4th edition. London: Saunders;2001:57-59

13 Perlas A, Chan VWS, Lupu CM, Mitsakakis N, Hanbidge A. Ultrasound assessment of gastric content and volume. Anesthesiology 2009;111(1):82-89

14 Singh S, Chowdhury V. Efficacy of high-resolution transabdominal sonography of the fluid-filled stomach in the evaluation of gastric carcinomas. Indian J Rad Imag 2005;15(4):421-426

15 Rapaccini GL, Aliotta A, Pompili M, et al. Gastric wall thickness in normal and neoplastic subjects: a prospective study performed by abdominal ultrasound. Gastrointest Radiol 1988;13(3):197-199

16 Megibow AJ, Duodenum. In: Megibow AJ, ed. Computed Tomography of the Gastrointestinal Tract. St. Louis: Mosby;1986: 175-216

17 Gossios KJ, Tsianos EV, Demou LL, et al. Use of water or air as oral contrast media for computed tomographic study of the gastric wall: comparison of the two techniques. Gastrointest Radiol 1991;16(4):293-297

18 Scatarige JC, DiSantis DJ. CT of the stomach and duodenum. Radiol Clin North Am 1989;27(4):687-706

19 Jayaraman MV, Mayo-Smith WW, Movson JS, Dupuy DE, Wallach MT. CT of the duodenum: an overlooked segment gets its due. Radiographics 2001;21(Spec No) :S147-S160
20 Horton KM, Fishman EK. Current role of CT in imaging of the stomach. Radiographics 2003;23(1):75-87

21 Fishman EK, Urban BA, Hruban RH. CT of the stomach: spectrum of disease. Radiographics 1996;16(5):1035-1054

22 Chou CK, Chen LT, Sheu RS, Wang ML, Jaw TS, Liu GC. MRI manifestations of gastrointestinal wall thickening. Abdom Imaging 1994;19(5):389-394

23 Lubienski A, Grenacher L, Reith W, Schipp A, Mechtersheimer G, Düx M. [MR imaging of gastric wall layers in vitro: correlation to the histologic wall structure]. RoFo Fortschr Geb Rontgenstr Nuklearmed 2002;174(4):490-494

24 Inui K, Nakazawa S, Yoshino J, et al. Endoscopic MRI: preliminary results of a new technique for visualization and staging of gastrointestinal tumors. Endoscopy 1995;27(7):480-485

25 Lei C, Huang L, Wang Y, Huang Y, Huang Y. Comparison of MRI and endoscope ultrasound detection in preoperative $\mathrm{T} / \mathrm{N}$ staging of gastric cancer. Mol Clin Oncol 2013;1(4):699-702

26 Nagler AK, Aslanian HR, Siddiqui UD. Endoscopic ultrasound and gastric lesions. J Clin Gastroenterol 2011;45(3):215-221

27 Saito N, Takeshita K, Habu H, Endo M. The use of endoscopic ultrasound in determining the depth of cancer invasion in patients with gastric cancer. Surg Endosc 1991;5(1):14-19

28 Farrell MB. Gastric emptying scintigraphy. J Nucl Med Technol 2019;47(2):111-119

29 Maughan RJ, Leiper JB. Methods for the assessment of gastric emptying in humans: an overview. Diabet Med 1996; 13(9 Suppl 5) :S6-S10 